\title{
The evolutionarily conserved factor Sus1/ENY2 plays a role in telomere length maintenance
}

Amparo Galán" ${ }^{\prime \prime}$ Encar García-Oliver ${ }^{1 "}$, Carme Nuño-Cabanes ${ }^{1.2}$ Linda Rubinstein $^{3}$, Martin Kupiec ${ }^{3}$ and Susana Rodríguez-Navarro',**

'Gene Expression and RNA Metabolism Laboratory. Centro de Investigación Príncipe Felipe, C/ E. Primo Yúfera 3, 46012 Valencia

${ }^{2}$ Gene Expression and RNA Metabolism Laboratory. Instituto de Biomedicina de Valencia, C/ Jaime Roig, 11, 46010 Valencia

${ }^{3}$ Department of Molecular Microbiology and Biotechnology, Tel Aviv University, Ramat Aviv 69978, Israel.

${ }^{\#}$ Co-first authors

*Corresponding author: E-mail srodriguez@cipf.es

ORCID: 0000-0001-7472-3111

Keywords: Telomere length, Sus1, H2B de-ubiquitination, SAGA, TREX-2, RSC 


\section{ABSTRACT}

Sus1 is a conserved protein involved in histone $\mathrm{H} 2 \mathrm{~B}$ de-ubiquitination and mRNA export from the nucleus in eukaryotes. Previous studies implicated Sus1 partners in genome integrity including telomere homeostasis. However, the implication of Sus1 in telomere maintenance remains largely unknown.

In this study, we found that yeast Sus1 interacts physically and genetically with factors involved in telomere maintenance and its absence leads to elongated telomeres. Deletion of several of Sus1's partners also leads to longer telomeres. Our results rule out a direct role for Sus1 in recruiting telomerase subunits to telomeres. However, we observe that deletion of SUSI leads to elongated telomeres even in the presence of mutations like $\operatorname{sem} 1 \Delta$, esc $2 \Delta$ and $\operatorname{rsc} 2 \Delta$, which cause telomere shortening. We find that $r s c 2 \Delta$ (short telomeres) have reduced levels of monoubiquitinated histone $\mathrm{H} 2 \mathrm{~B}$ at lysine $123(\mathrm{H} 2 \mathrm{BK} 123 \mathrm{ub})$, whereas sus $1 \Delta$ mutants or double mutants $\operatorname{sus} 1 \Delta r s c 2 \Delta$ exhibit longer telomeres and higher H2BK123ub'levels. These results suggest that Sus1 activity as a $\mathrm{H} 2 \mathrm{~B}$ de-ubiquitination modulator plays a role in negatively regulating telomere length. Our results provide solid evidence for a role of Sus1 in negatively regulating telomere length through the modulation of H2BK123 mono-ubiquitination and its interaction with the nuclear pore complex. 


\section{Introduction}

The transcription and mRNA export factor, Sus1, is physically and functionally associated with TREX-2 (TRanscription and EXport 2) -a nuclear pore-associated assembly involved in mRNA export- and with SAGA (Spt-Ada-Gen5 acetyltransferase) -a transcriptional coactivator that modifies histones- (Rodríguez-Navarro et al. 2004; Galan and Rodríguez-Navarro 2012). This physical interaction provides a mechanism that could perfectly integrate early transcriptional events, such as epigenetic modifications with downstream events at the nuclear pore complex (NPC) which occur during later steps of the mRNA life cycle. This specific feature of Sus1 as part of these complexes, makes its study an exceptional source in discovering novel functional connections between genome biology and mRNA export. Among these genome-dependent processes, different aspects of telomere biology have been shown to depend on TREX2 and SAGA.

Yeast cells provide an excellent model to study mechanisms that affect telomere biology (Askree et al. 2004; Simonicova et al. 2015; Machin et al. 2016; Millet and Makovets 2016; Gadaleta et al. 2016). Proteins located at the nuclear periphery in close proximity to TREX-2 have been implicated in genome integrity by protecting the telomeric repeats from recombination. For instance, it has been shown that eroded telomeres change their subnuclear location to the nuclear pore during the DNA damage response (Khadaroo et al. 2009). In addition to factors close to the nuclear periphery, several SAGA subunits have also been implicated in telomere homeostasis and ageing (Askree et al. 2004; Gatbonton et al. 2006; Shachar et al. 2008; Ungar et al. 2009; Atanassov et al. 2009). Work in yeast has shown that the SAGA subunit Ada2 binds the telomeres avoiding the spreading of heterochromatin into 
subtelomeric regions (Jacobson and Pillus 2009). Further studies have focused on studying factors that belongs to the SAGA de-ubiquitination module (DUBm), of which Sus1 is part. The DUBm is composed by Ubp8, Sgf11, Sgf73 and Sus1 (Ingvarsdottir et al. 2005; Lee et al. 2005; Köhler et al. 2006). All four proteins are required for full de-ubiquitination of histone H2B mono-ubiquitinated at lysine 123 (H2BK123ub deubiquitinase activity) both in vivo and in vitro (Köhler et al. 2010; Samara et al. 2010; Morgan et al. 2016). The DUBm is attached to SAGA by its subunit Sgf73, which together with Sus 1 links SAGA with TREX2 and mRNA export (Köhler et al. 2008; Lee et al. 2009; Pascual-García and RodríguezNavarro 2009; Rodríguez-Navarro 2009). Studies focused on DUBm subunits, as for instance Sgf73, demonstrated that its deletion dramatically extends replicative life span (RLS) in a Sir2 dependent manner (McCormick et al. 2014) and reduces rDNA circle retention (DenothLippuner et al. 2014). rDNA circle retention is one important factor correlated with ageing in yeast (Sinclair and Guarente 1997). Interestingly, TREX-2, which is the other complex in which Sus1 resides, is also required for the asymmetric segregation of these rDNA circles during yeast ageing (Denoth-Lippuner et al. 2014). More recent work has shown that the replicative lifespan-extending deletion of $S G F 73$ results in altered ribosomal gene expression (Mason et al. 2017). These findings suggest that Sgf73 target genes are integral to pathways of aging regulation in yeast. The enzymatic activity of the DUBm is achieved by Ubp8, which has also been implicated in maintaining $\mathrm{H} 2 \mathrm{~B}$ monoubiquitination levels (H2BK123ub') at the telomeres (Wu, et al. 2017). Although this role is more effectively performed by Ubp10 (Schulze et al. 2011) and Ubp6 (Qin et al. 2009), a very recent report suggests that inactivation of Ubp8 promotes telomere extension in yeast (Wu, et al. 2017). The DUBm, as most of SAGA subunits, is evolutionary conserved in terms of composition and cellular 
functions (Rodríguez-Navarro 2009; Koutelou et al. 2010; Spedale et al. 2012; Wang and YR Dent 2014). Therefore, it is not surprising that USP22 (hUbp8) has been also shown to function in telomere maintenance in human cells by regulating the turnover of a shelterin protein (TRF1) (Atanassov, et al. 2009b).

Despite the evidence showing that functional interactors of Sus1, both from SAGA and the NPC, participate in telomere length homeostasis, Sus1's role in this pathway is poorly described. Previously, we found a very strong genetic interaction between Sus1 and Asf1 (Anti-Silencing Factor 1), which is a nucleosome assembly factor involved in chromatin assembly and disassembly and an anti-silencing protein that causes de-repression of silent loci when overexpressed (Schwabish and Struhl 2006). We reported that Sus1, together with TREX-2, functionally interacts with Asf1 (Pamblanco et al. 2014), which has also been linked to the alternative lengthening of telomeres (ALT) pathway of mammalian chromosome-end maintenance (O'sullivan et al. 2014). Furthermore, a novel role for yeast Asf1 and H2BK123ub in the epigenetic silencing of the silent mating loci has been proposed (Wu et al. 2017a), connecting the functional interactions of Sus1 with the mechanisms that mediate the maintenance of chromatin at telomeres and silent loci.

In this study, we found that yeast Sus1 interacts physically and genetically with factors involved in telomere maintenance. We show that sus $1 \Delta$ strains exhibit elongated telomeres, similar to its partners in SAGA and TREX-2 complexes. This phenotype is epistatic to other mutations affecting SAGA and RSC subunits. We discuss a possible role of H2BK123 mono-ubiquitination dependent on Sus1, SAGA and TREX-2 in telomere length maintenance. 


\section{Results and Discussion}

\section{Sus1 interacts physically and genetically with genes affecting telomere length}

In a previous study, we showed, by SUS1-TAP purification, that in addition to SAGA and TREX-2 components, Sus1 was co-enriched with other proteins (Cuenca-Bono et al. 2010; Pamblanco et al. 2014). These findings extended possible connections between Sus1 and genes involved in chromatin and RNA metabolism. Notably, LC-MS/MS analyses from these Sus1-TAP purified complexes also identified several proteins functionally linked to telomere homeostasis. Among the factors identified by LC-MS/MS co-purifying with Sus1, we validated, by co-IP, the co-purification of Yku80, Upf2, Mlp2 and Rif1 with Sus1. Whereas Rif1 and the Ku heterodimer (composed of Yku70 and Yku80) bind telomeres, the other two proteins are known regulators of telomere length (Grandin et al. 2000; Hediger et al. 2002; Levy and Blackburn 2004; Addinall et al. 2011). First, Sus1 was TAP tagged and purified in cells co-expressing Yku80-PK or Upf2-PK respectively. As shown in Fig. 1a, Yku80-PK and Upf2-PK were detected after Sus1-TAP purification. Similar results were obtained when Mlp2 and Rif1 tagged with MYC were used as bait proteins in the co-IP experiments. In both cases, Sus1 co-purified after immunoprecipitation with MYC antibodies (Fig. 1b). These experiments confirmed that Sus1 co-purifies with several factors involved in telomere maintenance and homeostasis.

Simple organisms such as budding and fission yeast have contributed enormously to our understanding of telomere biology (Simonicova et al. 2015; Millet and Makovets 2016; Gadaleta et al. 2016). A number of studies in Saccharomyces cerevisiae identified mutants 
that affect telomere length. Genome-wide analyses annotated a set of close to 500 TelomereLength-Mutants (tlm) (Gatbonton et al. 2006; Shachar et al. 2008; Yosef et al. 2009; Ungar et al. 2009). About half of these mutants exhibit longer than wild type, and half shorter than wild type phenotypes. To support the physical interaction between Sus1 and genes involved in telomere homeostasis, we investigated the genetic network of sus $1 \Delta$ obtained from the BIOGRID database (http://www.thebiogrid.org/index.php), which consisted of 319 genes (Table S1), and compared it with genes annotated as "TLM" genes (466 genes, Table S1). These analyses revealed that out of the 319 genetic interactors of sus $1 \Delta, 54$ genes corresponded to $T L M$ genes (Fig. 2a). This represents $17 \%$ of the sus $1 \Delta$ genetic network. A Fischer test used to calculate the statistical significance of this proportion revealed that this enrichment was significant $(\mathrm{P}-\mathrm{value}<0.00005)$. Although the percentage of genes leading to short telomeres was higher in the sus $1 \triangle T L M$ network (Fig. 2b), the difference between short or long $T L M$ mutants was not significant $(\mathrm{P}$-value $<0.1)$.

Next, we validated some of these predicted genetic interactions by creating double mutants bearing a deletion of SUS1 and deletion of genes selected from Fig. 2a. As shown in Fig. 2c, deletion of MLP2 in sus $1 \Delta$ cells exacerbated the growth defect of each single mutant at $30^{\circ} \mathrm{C}$ and $37^{\circ} \mathrm{C}$. Notably the strongest effect was observed when SUS1 was deleted together with $R S C 2$, which is a component of the RSC chromatin remodeling complex (Fig. 2c). This complex has been shown to affect the function of nuclear pores and the nuclear envelope (Titus, et al. 2010). In addition, mutations in RSC subunits were found to affect telomere length maintenance (Askree et al. 2004; Ungar et al. 2009) and the repair of double-stranded breaks (Shim et al. 2005). 


\section{Deletion of $S U S 1$ leads to elongated telomeres in yeast}

Our data, together with the fact that depletion of some components of hSAGA DUBm provokes telomere-induced foci and telomere elongation (Atanassov, et al. 2009a), prompted us to analyze the length of telomeres in a sus $1 \Delta$ strain. Fig. 3a shows that telomeres were slightly longer in sus $1 \Delta$ strain compared to the wild-type (compare lanes 1 and 2). This suggests that Sus1 acts as a negative regulator of telomere length.

Long telomeres could be a consequence of deregulation of telomerase activity. Telomerase subunits are targeted to telomeres following an intricate mechanism [Reviewed in (Kupiec 2014)]. For instance, telomerase components Est2/Tlc1 associate with telomeres in both $\mathrm{G}_{1}$ and $\mathrm{S}$ phases through different mechanisms (Chan et al. 2008; Tong et al. 2011). In order to address whether the elongated phenotype observed for SUS1 deletion resulted from a deregulation of telomerase tethering to telomeres, the recruitment of telomerase components Est 1 and Est 2 to several telomeric regions was analyzed in sus $1 \Delta$ mutant cells and compared to wild-type cells. In these experiments, we monitored binding of the telomerase subunits Est1 and Est2 to three different telomeric regions: 7L, 15L and Y'. Previous experiments reported robust binding of these proteins to telomeres. Fig. $3 \mathrm{~b}$ shows that binding of Est1 and Est2 to telomeric regions, 7L, 15L and Y' was independent of Sus 1. We thus conclude that in non-synchronized cells, Sus1 does not play a direct role in recruiting telomerase subunits to telomeres. As cited above, previous experiments reported robust binding of these proteins to telomeres during late-S and G2/M phase. In this regard, we can not exclude the possibility the Sus1 contributes to telomerase recruitment under specific 
stages during the different cell cycle phases. Further work is required to ascertain this interesting possibility.

\section{Additional factors in TREX-2 and SAGA affect telomere length}

Two mutants affecting nuclear pore-associated proteins, sem $1 \Delta$ and $\operatorname{esc} 2 \Delta$, show slightly shortened telomeres. These two mutants are defective for factors that have been shown to affect DNA replication, recombination and repair. Sem1, as part of the TREX-2 complex, interacts with the NPC and participates in DNA replication (Bermejo et al. 2011), and in the prevention of transcription-associated genome instability (González-Aguilera et al. 2008). Previous work also demonstrated that Sem1 binds to Ubp6 which also deubiquitinates H2B at telomeres (Qin, et al. 2009). Simultaneous deletion of SEMI and UBP6 blocks $\mathrm{H} 2 \mathrm{~B}$ de-ubiquitination, resulting in derepression of telomeric genes by dissociation of the SIR complexes (Qin, et al. 2009). Esc2, as part of the RENi complex (Rad60, Esc2, Nip45) is necessary for relocation to the NPC of heterochromatic Double Strand Breaks (DBSs) (Chiolo et al. 2011) (Ryu et al. 2016). As such, Esc2 influences multiple replication-associated recombination processes in Saccharomyces cerevisiae (Choi et al. 2010).

To study the interactions between Sus1 and these factors, double mutants $\operatorname{sus} 1 \Delta \operatorname{sem} 1 \Delta$ and $\operatorname{sus} 1 \Delta e s c 2 \Delta$ were created. Notably, the experiments revealed that the phenotype of the susld mutation was epistatic since the slightly short $t l m$ phenotypes provoked by deletion of SEM1 and ESC2 were completely suppressed by SUS1 deletion in these double mutants, which showed the slight elongated telomere phenotype of sus $1 \Delta$ (Fig. 3a). 
The epistatic effect of sus $1 \Delta$ suggests that the short telomeres observed in the single $\operatorname{sem} 1 \Delta$ and $\operatorname{esc} 2 \Delta$ mutants are a consequence of Sus1 function. We speculated that this is mediated by the role of Sus1 at the NPC through the TREX-2 complex. In this model, Sus1 plays a role in the tethereing of eroded telomeres to the NPC. The absence of Sem1 and Esc2 slightly affects telomere length, but only if they are tethered by Sus1 to the NPC. To experimentally support this model, we also investigated the length of telomeres in other two mutants of TREX-2; $\operatorname{sac} 3 \Delta$ and thpl $\Delta$. As shown in Fig. 3c (lanes $6 \&$ 7) telomeres were slightly longer in both TREX-2 mutants compared to the wild-type. Taken together these results suggest that the elongated telomeres phenotype observed for sus $1 \Delta$ could be a consequence of its role at the NPC.

To study to what extent SUSI deletion is able to suppress short telomere phenotypes of factors known to affect NPC-related functions, we chose the $R S C 2$ gene, since strains simultaneously deleted for SUS1 and RSC2 exhibited a very strong synthetic lethality (Fig. 2c). RSC2 is part of the RSC complex (Wong et al. 2002) which affects the nuclear pore function (Titus et al. 2010) and its deletion shortens significantly telomere length (Askree et al. 2004). More recently, through a telomere length kinetics assay (TELKA), the $\operatorname{rsc} 2 \Delta$ mutant was classified into a functional group of mutants that exhibited mild exponential shortening of telomeres (Rubinstein et al. 2014). Remarkably, deletion of SUS1 in a rsc2A mutant strain also resulted in telomere elongation, a phenotype similar to that of the single sus $1 \Delta$ mutant (Fig. 3a, lanes $7 \&$ 8). These results suggest that, similarly to Sem1 and Esc2, the phenotype observed in $r s c 2 \Delta$ cells requires Sus1-mediated tethering to the NPC.

In addition to its association with NPC proteins, Sus1 is also present in the SAGA DUBm and, as summarized in the introduction, SAGA and DUBm factors have been 
involved in telomere maintenance in humans (Atanassov, et al. 2009b). We therefore analyzed deletions of the yeast counterparts of human DUBm components like Sgf11, Sgf73 and Ubp8. As shown in Fig. 3c (see lanes 8, 9 and 10), telomeres were also slightly longer compared to the wild-type for all DUBm mutant. This is consistent with a recent study demonstrating that inactivation of both Ubp8 and Ubp10 promotes telomere elongation (Wu et al.2017b). Since the phenotype of elongated telomeres are shared by all DUBm, our results suggest that Ubp8 activity takes place in the context of the DUBm as a complete module, by playing a relevant role in telomere homeostasis in yeast.

The deubiquitinase activity of the DUBm is important, since H2B monoubiquitination is a central epigenetic modification required for different cellular processes (Chandrasekharan et al. 2010), including transcriptional regulation, DNA replication, embryonic stem cell differentiation (Karpiuk et al. 2012) and autophagy (Chen et al. 2017; Hung et al. 2017; Huang et al. 2017). H2Bub mediated by Rad6-Bre1 has been also studied at telomeres showing that the absence of this epigenetic mark leads to loss of telomeric silencing (Sun and Allis 2002). It is clear than the role of Sus1 via its interaction with the NPC (mediated by TREX-2) and in H2B de-ubiquitination (mediated by SAGA) places this factor in a central hub likely regulating chromatin modifications at the NPC with important implications in telomere homeostasis.

\section{Short telomere mutants $r s c 2 \Delta$ and $e s t 1 \Delta$ show low levels of H2B monoubiquitination}

The functional implications of $\mathrm{H} 2 \mathrm{~B}$ monoubiquitination in telomere homeostasis has been the subject of intense study in recent years. However, a precise correlation between telomere length and global H2B monoubiquitination levels is unknown. The data presented in this study, together with recent work in humans and yeast (Armour et al. 2013; Atanassov 
et al. 2016; Wu et al. 2017b) suggested that a normal cycle of ubiquitination and deubiquitination of H2BK123, as has been found for other proteins (Chu et al. 2016; Jiang 2016; Nostramo and Herman 2016), influences telomere length. Thus, we decided to investigate whether mutants with short telomeres, such as $r s c 2 \Delta$, resulted in a depletion of H2BK123ub. Whole cell extracts from wt, sus $1 \Delta$ and $\operatorname{rsc} 2 \Delta$ were obtained and the levels of H2BK123ub were measured by Western-blot. Quantification of three independent experiments are shown in Fig. 4a. Deletion of $R S C 2$, which leads to short telomeres, provoked a significant reduction in the global levels of H2BK123ub (Fig. 4a). As a control to test whether any strain with short telomeres shows reduced levels of $\mathrm{H} 2 \mathrm{BK} 123 \mathrm{ub}^{\prime}$ we also measured its levels in a est $1 \Delta$ strain (Est1 encodes a subunit of telomerase). Although slightly reduced, the levels were not statistically different from those of the wt strain (Fig. 4b). Interestingly, deletion of SUS1 in any of the single mutants $\operatorname{rsc} 2 \Delta$ or est $1 \Delta$ resulted in a sus $1 \Delta$ epistatic phenotype, with a high level of $\mathrm{H} 2 \mathrm{BK} 123 \mathrm{ub}$ in the cell extract (Fig. 4c). Thus, a clear correspondence is observed between the level of $\mathrm{H} 2 \mathrm{~B}$ monoubiquitination and the telomeric phenotype of single and double mutants, suggesting that Sus1 activity as a DUB modulator plays a role in negatively regulating telomere length. Our results are consistent with a model in which RSC activity negatively regulates the de-ubiquitination activity of Sus1. When RSC activity is absent, Sus1, as part of the DUBm, deubiquitinates H2BK123 leading to shorter telomeres. Deletion of Sus1 causes an accumulation of H2BK123ub that suppresses the short telomere phenotype and elongates telomeres irrespective of other mutations such as $\operatorname{rsc} 2 \Delta$, $\operatorname{sem} 1 \Delta$ or esc $2 \Delta$. Future studies should determine whether $\mathrm{H} 2 \mathrm{BK} 123 \mathrm{ub}$ de-ubiquitination is instrumental in tethering telomeres to nuclear pores, or whether nuclear pore localization is a pre-requisite for the modification. Our results suggest 
that the ubiquitination status of histones at telomeres affects, directly or indirectly, telomere length homeostasis. Further experiments will be directed at defining the exact molecular mechanism of this regulation.

In summary, supportive of a functional interaction between Sus1 and the telomere homeostasis pathway, we demonstrate that Sus1 deletion leads to longer telomeres. Notably, the sus $1 \Delta$ phenotype persisted in mutants with short telomeres like sem $1 \Delta$ and $r s c 2 \Delta$ mutants, underscoring a potential role for $\mathrm{H} 2 \mathrm{BK} 123 \mathrm{ub}$ in maintaining normal telomere length in yeast. Our experiments rule out the possibility that this modification directly affects telomerase recruitment, and suggest a more indirect effect, perhaps by affecting the coordination between DNA replication and telomere addition at the NPC. Since Sus1 is an evolutionary conserved factor (ENY2, in human cells) this study opens the possibility of a novel role for Sus1/ENY2 in telomere maintenance in higher eukaryotes.

\section{Materials and methods}

\section{Yeast strains, DNA recombinant work, and microbiological techniques}

Yeast strains used in this study are listed in Table S2 and described in the Supplemental Material, along with the antibodies and PCR primers employed (Table S3). Microbiological techniques and yeast plasmid transformation were done essentially as described previously (Rodriguez-Navarro, 2004) . Chromosomal integration of TAP, PK and MYC was performed as described previously (Longtine et al. 1998; Gavin et al. 2002). For gene disruptions, the indicated gene was deleted by high efficiency transformation by using either a PCR product amplified from the KanMX4 plasmid pRS400 or the HIS3 plasmid pFA6a. All deletions and genomically tagged strains were confirmed by PCR analysis and/or 
Western blot analysis. Strains were grown under standard conditions. For growth analysis, yeast cells were diluted to $0.5 \mathrm{OD}_{600}$, and serial dilutions (1:10) were spotted onto YPD and incubated at various temperatures.

\section{TAP purifications, immunoprecipitations and Western blot analysis}

Purification of Sus1-TAP Yku80-PK and Sus1-TAP Upf1-PK strains was performed as described previously (Rodriguez-Navarro, 2004) . Sus1-TAP fusion protein and associated proteins were recovered from cell extracts by affinity selection on an IgG matrix. After washing, the TEV protease is added to release the bound material. The eluate is incubated with calmodulin-coated beads in the presence of calcium. This second affinity step is required to remove the TEV protease as well as traces of contaminants remaining after the first affinity selection. After washing, the bound material is released with EGTA. This enriched fraction is called Calmoduline eluate. Calmoduline eluates from the TAP-purified complexes were analyzed by SDS-PAGE gels. Western analysis of the Calmoduline eluates was performed using anti-PK and anti-TAP antibody according to standard procedures.

Immunoprecipitation of Sus1-TAP Mlp2-MYC and Sus1-TAP Rif1-MYC was performed as follows: $50 \mathrm{~mL}$ of cells expressing Sus1-TAP were grown on rich medium to a $0.5 \mathrm{OD}_{\text {бо }}$. Cells were harvested, washed with water, and resuspended in $250 \mu \mathrm{L}$ of lysis buffer (50 mM HEPES-KOH at pH 7.5, 140 mM NaC1, 1 mM EDTA, 10\% glycerol, 0.5\% NP-40, $1 \mathrm{mM}$ PMSF, protease inhibitors). An equal volume of glass beads was added. Breakage was achieved by four pulses of vortexing during 1 minute at $4{ }^{\circ} \mathrm{C}$. The extracts clarified were immunoprecipitated during 60 minutes at $4{ }^{\circ} \mathrm{C}$ using anti-Myc antibody. The immunoprecipitates were washed three times for 10 minutes with $1 \mathrm{~mL}$ of lysis buffer and 
subsequently resuspended in $50 \mu \mathrm{L}$ of SDSPAGE sample buffer. Western analysis was performed using using anti-MYC and anti-TAP antibodies according to standard procedures.

\section{Chromatin immunoprecipitation}

ChIP analysis was performed as described elesewhere (Fisher et al. 2004) with slight modifications. Briefly, all immunoprecipitates were washed with $0.025 \%$ (w/v) SDS, ChiP DNA Clean \& Concentrator (Zymo) was used for chromatin purification, and Clontech antimyc monoclonals was used for immunoprecipitation. Quantitation of immunoprecipitated DNA was performed via real-time PCR analysis, using a Lightcycler 480 detection system equipment (Roche Diagnostics). Primers were specific for amplification of $\mathrm{Y}^{\prime}, 151$ and 7L telomeric region (Table S3). Enrichment was expressed related to the internal control ARO1. Each experiment was repeated at least three times and statistics were calculated using the ANOVA test.

\section{Southern blots}

Teloblots were carried out as previous ly described (Rubinstein, et al. 2014). A Saccharomyces cerevisiae-specific telomeric probe and a size marker were used as probes. The size-control probe is a specific region of chromosome II (positions 558490 to 559790) that detects two bands in the XhoI digested genomic DNA (2044 and 779bp long).

\section{Figure Captions}

\section{Figure 1: Sus1 interacts physically with genes affecting telomere length}


TAP purification of Sus1-TAP from Yku80-PK or Upf2-PK tagged cells was performed. The presence of Yku80, Upf2 and Sus1 within purified eluates was monitored by western blot using anti-PK and anti-TAP antibodies (IPs). Input levels were detected using anti-PK and anti-TAP antibodies (INPUTs). Yku80-PK or Upf2-PK strains without the TAP tagged were

used as a control (a) Mlp2-MYC or Rif1-MYC strains containning Sus1-TAP were immunoprecipitated from WCEs. The presence of Sus1 and the levels of Mlp2 and Rif1 bait proteins were monitored in immunoprecipitated eluates (IPs) and inputs (INPUTs) using antiTAP and anti-MYC antibodies (b).

\section{Figure 2: Sus1 interacts genetically with genes affecting telomere length}

Venn diagramm showing an overlap between the genetic network of sus $1 \Delta$ and TelomereLength-Mutants (tlm)- annotated genes. A Fischer test was used to calculate the P-value of the association (a) Distribution of long and short telomere phenotype among the 54 tlm-genes from the genetic network of sus $1 \Delta$ and global $t l m$ distribution. A Fischer test was used to calculate the P-value of the association (b) Serial ten-fold dilutions of wild-type, sus $1 \Delta$, $m l p 2 \Delta$ and $\operatorname{rsc} 2 \Delta$ single mutants and the same mutants combined with a deletion of SUS1 were spotted onto YPD plates. Cells were grown for $48 \mathrm{~h}$ at $30^{\circ} \mathrm{C}$ and $37^{\circ} \mathrm{C}(\mathbf{c})$.

\section{Figure 3: Deletion of $S U S 1$ leads to elongated telomeres in yeast}

Southern blot of telomeres of single- and double mutants. DNA was digested with XhoI before being run in a gel, transferred to a membrane and probed with a telomeric probe (a). Chromatin Immunoprecipitation (ChIP) analysis showing the recruitment levels of Est1 and Est2 proteins to $\mathrm{Y}^{\prime}$-containing, $7 \mathrm{~L}$ and $15 \mathrm{~L}$ telomeres in wild-type and sus $1 \Delta$ mutant. The 
non-telomeric $A R O 1$ locus was used to normalize the relative levels. Each experiment was repeated at least three times and statistics were calculated using the ANOVA test (b). Southern blot of telomeres of single- and double mutants. DNA was digested with XhoI before being run in a gel, transferred to a membrane and probed with a Y' probe (c).

Figure 4: Deletion of $R S C 2$ and $E S T 1$ affects normal levels of H2B monoubiquitination Quantification of Western-blot showing total H2BK123ub levels compared to total H2B and the internal control $P G K 1$ in WT, sus $1 \Delta$ and $r s c 2 \Delta$. Each experiment was repeated at least three times and statistics were calculated using the ANOVA test (a). (b) as in (a) for WT, $\operatorname{sus} 1 \Delta$ and est $1 \Delta$. (c) as in (a) for WT, sus $1 \Delta$, sus $1 \Delta \operatorname{rsc} 2 \Delta$ and $\operatorname{sus} 1 \Delta e s t 1 \Delta$. Quantification analysis (left panel) and a representative image of the Western-blot (right panel).

Acknowledgements We would like to acknowledge Dr. Virginia Zakian from Princeton University, for her kind donation of LKG236 (Est1-Myc) and LKG239 (Est2-Myc) strains used in this study. We thank present and past members of the Rodriguez-Navarro and Kupiec lab for ideas, encouragement and support.

\section{Funding}

This work was supported by FEDER 2014-2020 and Ministerio de Economia y Competitividad (MINECO) of Spain (BFU2011-23418 and BFU2014-57636-P to S.R.-N.), by Generalitat Valenciana of Spain (ACOMP/2014/061 and ACOMP/2015/096 to S.R-N., C. N-C was supported by the PROMETEO program (PROMETEO/2016/093). Work in 
MK's lab was supported by the Israel Science Fund and the Israel Cancer Research Foundation (ICRF).

\section{References}

Addinall SG, Holstein EM, Lawless C, et al (2011) Quantitative fitness analysis shows that NMD proteins and many other protein complexes suppress or enhance distinct telomere cap defects. PLoS Genet 7:e1001362. doi: 10.1371/journal.pgen.1001362

Armour SM, Bennett EJ, Braun CR, et al (2013) A High-Confidence Interaction Map Identifies SIRT1 as a Mediator of Acetylation of USP22 and the SAGA Coactivator Complex. Mol Cell Biol 33:1487-1502. doi: 10.1128/MCB.00971-12

Askree SH, Yehuda T, Smolikov S, et al (2004) A genome-wide screen for Saccharomyces cerevisiae deletion mutants that affect telomere length. Proc Natl Acad Sci USA 101:8658-8663. doi: 10.1073/pnas.0401263101

Atanassov BS, Evrard YA, Multani AS, et al (2009) Gcn5 and SAGA regulate shelterin protein turnover and telomere maintenance. Mol Cell 35:352-364. doi: 10.1016/j.molcel.2009.06.015

Atanassov BS, Mohan RD, Lan X, et al (2016) ATXN7L3 and ENY2 Coordinate Activity of Multiple H2B Deubiquitinases Important for Cellular Proliferation and Tumor Growth. Mol Cell 1-15. doi: 10.1016/j.molcel.2016.03.030

Bermejo R, Capra T, Jossen R, et al (2011) The replication checkpoint protects fork stability by releasing transcribed genes from nuclear pores. Cell 146:233-246. doi: 10.1016/j.cell.2011.06.033

Chan A, Boulé J-B, Zakian VA (2008) Two pathways recruit telomerase to Saccharomyces cerevisiae telomeres. PLoS Genet 4:e1000236. doi: 10.1371/journal.pgen.1000236

Chandrasekharan MB, Huang F, Sun Z-W (2010) Histone H2B ubiquitination and beyond: Regulation of nucleosome stability, chromatin dynamics and the trans-histone H3 methylation. epigenetics. doi: 10.4161/epi.5.6.12314

Chen S, Jing Y, Kang X, et al (2017) Histone H2B monoubiquitination is a critical epigenetic switch for the regulation of autophagy. Nucleic Acids Res 45:1144-1158. doi: 10.1093/nar/gkw1025

Chiolo I, Minoda A, Colmenares SU, et al (2011) Double-strand breaks in heterochromatin 
move outside of a dynamic HP1a domain to complete recombinational repair. Cell 144:732-744. doi: 10.1016/j.cell.2011.02.012

Choi K, Szakal B, Chen Y-H, et al (2010) The Smc5/6 complex and Esc2 influence multiple replication-associated recombination processes in Saccharomyces cerevisiae. Mol Biol Cell 21:2306-2314. doi: 10.1091/mbc.E10-01-0050

Chu X-L, Feng M-G, Ying S-H (2016) Qualitative ubiquitome unveils the potential significances of protein lysine ubiquitination in hyphal growth of Aspergillus nidulans. Curr Genet 62:191-201. doi: 10.1007/s00294-015-0517-7

Cuenca-Bono B, García-Molinero V, Pascual-García P, et al (2010) A novel link between Sus1 and the cytoplasmic mRNA decay machinery suggests a broad role in mRNA metabolism. BMC Cell Biol 11:19. doi: 10.1186/1471-2121-11-19

Denoth-Lippuner A, Krzyzanowski MK, Stober C, Barral Y (2014) Role of SAGA in the asymmetric segregation of DNA circles during yeast ageing. eLife. doi: 10.7554/eLife. 03790

Fisher TS, Taggart AKP, Zakian VA (2004) Cell cycle-dependent regulation of yeast telomerase by Ku. Nat Struct Mol Biol 11:1198-1205. doi: 10.1038/nsmb854

Gadaleta MC, Gonzalez-Medina A, Noguchi E (2016) Timeless protection of telomeres. Curr Genet 62:725-730. doi: 10.1007/s00294-016-0599-x

Galan A, Rodríguez-Navarro S (2012) Sus1/ENY2: a multitasking protein in eukaryotic gene expression. Critical Reviews in Biochemistry and Molecular Biology 47:556-568. doi: 10.3109/10409238.2012.730498

Gatbonton T, Imbesi M, Nelson M, et al (2006) Telomere length as a quantitative trait: genome-wide survey and genetic mapping of telomere length-control genes in yeast. PLoS Genet 2:e35. doi: 10.1371/journal.pgen.0020035

Gavin A-C, Bösche M, Krause R, et al (2002) Functional organization of the yeast proteome by systematic analysis of protein complexes. Nature 415:141-147. doi: $10.1038 / 415141 \mathrm{a}$

González-Aguilera C, Tous C, Gómez-González B, et al (2008) The THP1-SAC3-SUS1CDC31 complex works in transcription elongation-mRNA export preventing RNAmediated genome instability. Mol Biol Cell 19:4310-4318. doi: 10.1091/mbc.E08-040355

Grandin N, Damon C, Charbonneau M (2000) Cdc13 cooperates with the yeast Ku proteins and Stn1 to regulate telomerase recruitment. Mol Cell Biol 20:8397-8408.

Hediger F, Neumann FR, Van Houwe G, et al (2002) Live imaging of telomeres: yKu and Sir proteins define redundant telomere-anchoring pathways in yeast. Curr Biol 12:2076-2089. 
Huang X, Yang L, Cai F-F, et al (2017) Autophagy-related protein ATG5 regulates histone H2B mono-ubiquitylation by translational control of RNF20. J Genet Genomics. doi: 10.1016/j.jgg.2017.08.004

Hung S-H, Wong RP, Ulrich HD, Kao C-F (2017) Monoubiquitylation of histone H2B contributes to the bypass of DNA damage during and after DNA replication. Proc Natl Acad Sci USA 114:E2205-E2214. doi: 10.1073/pnas.1612633114

Ingvarsdottir K, Krogan NJ, Emre NCT, et al (2005) H2B ubiquitin protease Ubp8 and Sgf11 constitute a discrete functional module within the Saccharomyces cerevisiae SAGA complex. Mol Cell Biol 25:1162-1172. doi: 10.1128/MCB.25.3.11621172.2005

Jacobson S, Pillus L (2009) The SAGA subunit Ada2 functions in transcriptional silencing. Mol Cell Biol 29:6033-6045. doi: 10.1128/MCB.00542-09

Jiang Y (2016) Regulation of TORC1 by ubiquitin through non-covalent binding. Curr Genet 62:553-555. doi: 10.1007/s00294-016-0581-7

Karpiuk O, Najafova Z, Kramer F, et al (2012) The histone H2B monoubiquitination regulatory pathway is required for differentiation of multipotent stem cells. Mol Cell 46:705-713. doi: 10.1016/j.molcel.2012.05.022

Khadaroo B, Teixeira MT, Luciano P, et al (2009) The DNA damage response at eroded telomeres and tethering to the nuclear pore complex. Nat Cell Biol 11:980-987. doi: $10.1038 /$ ncb1910

Koutelou E, Hirsch CL, Dent SY (2010) Multiple faces of the SAGA complex. Curr Opin Cell Biol. doi: 10.1016/j.ceb.2010.03.005

Köhler A, Pascual-García P, Llopis A, et al (2006) The mRNA export factor Sus1 is involved in Spt/Ada/Gen5 acetyltransferase-mediated H2B deubiquitinylation through its interaction with Ubp8 and Sgf11. Mol Biol Cell 17:4228-4236. doi:

10.1091/mbc.E06-02-0098

Köhler A, Schneider M, Cabal GG, et al (2008) Yeast Ataxin-7 links histone deubiquitination with gene gating and mRNA export. Nat Cell Biol 10:707-715. doi: $10.1038 /$ ncb 1733

Köhler A, Zimmerman E, Schneider M, et al (2010) Structural basis for assembly and activation of the heterotetrameric SAGA histone H2B deubiquitinase module. Cell 141:606-617. doi: 10.1016/j.cell.2010.04.026

Kupiec M (2014) Biology of telomeres: lessons from budding yeast. FEMS Microbiol Rev $38: 144-171$.

Lee K, Florens L, Swanson S, et al (2005) The deubiquitylation activity of Ubp8 is dependent upon Sgf11 and its association with the SAGA complex. Mol Cell Biol 
25:1173-1182.

Lee KK, Swanson SK, Florens L, et al (2009) Yeast Sgf73/Ataxin-7 serves to anchor the deubiquitination module into both SAGA and Slik(SALSA) HAT complexes.

Epigenetics Chromatin 2:2. doi: 10.1186/1756-8935-2-2

Levy DL, Blackburn EH (2004) Counting of Rif1p and Rif2p on Saccharomyces cerevisiae telomeres regulates telomere length. Mol Cell Biol 24:10857-10867. doi:

10.1128/MCB.24.24.10857-10867.2004

Longtine M, McKenzie A, Demarini D, et al (1998) Additional modules for versatile and economical PCR-based gene deletion and modification in Saccharomyces cerevisiae. Yeast 14:953-961.

Machin F, Quevedo O, Ramos-Perez C, Garcia-Luis J (2016) Cdc14 phosphatase: warning, no delay allowed for chromosome segregation! Curr Genet 62:7-13. doi: 10.1007/s00294-015-0502-1

Mason AG, Garza RM, McCormick MA, et al (2017) The replicative lifespan-extending deletion of SGF73 results in altered ribosomal gene expression in yeast. Aging Cell 16:785-796. doi: 10.1111/acel.12611

McCormick MA, Mason AG, Guyenet SJ, et al (2014) The SAGA Histone Deubiquitinase Module Controls Yeast Replicative Lifespan via Sir2 Interaction. CellReports 1-10. doi: 10.1016/j.celrep.2014.06.037

Millet C, Makovets S (2016) Aneuploidy as a mechanism of adaptation to telomerase insufficiency. Curr Genet 62:557-564. doi: 10.1007/s00294-015-0559-X

Morgan MT, Haj-Yahya M, Ringel AE, et al (2016) Structural basis for histone H2B deubiquitination by the SAGA DUB module. Science 351:725-728. doi: $10.1126 /$ science.aac5681

Nostramo R, Herman PK (2016) Deubiquitination and the regulation of stress granule assembly. Curr Genet 62:503-506. doi: 10.1007/s00294-016-0571-9

O'sullivan RJ, Arnoult N, Lackner DH, et al (2014) Rapid induction of alternative lengthening of telomeres by depletion of the histone chaperone ASF1. Nat Struct Mol Biol 21:167-174. doi: 10.1038/nsmb.2754

Pamblanco M, Oliete-Calvo P, García-Oliver E, et al (2014) Unveiling novel interactions of histone chaperone Asf1 linked to TREX-2 factors Sus1 and Thp1. Nucleus (Austin, Tex) 5:247-259. doi: 10.4161/nucl.29155

Pascual-García P, Rodríguez-Navarro S (2009) A tale of coupling, Sus1 function in transcription and mRNA export. RNA Biol 6:141-144.

Qin S, Wang Q, Ray A, et al (2009) Sem1p and Ubp6p orchestrate telomeric silencing by 
modulating histone $\mathrm{H} 2 \mathrm{~B}$ ubiquitination and $\mathrm{H} 3$ acetylation. Nucleic Acids Res 37:1843-1853. doi: 10.1093/nar/gkn1049

Rodríguez-Navarro S (2009) Insights into SAGA function during gene expression. EMBO Rep 10:843-850. doi: 10.1038/embor.2009.168

Rodríguez-Navarro S, Fischer T, Luo M-J, et al (2004) Sus1, a functional component of the SAGA histone acetylase complex and the nuclear pore-associated mRNA export machinery. Cell 116:75-86.

Rubinstein L, Ungar L, Harari Y, et al (2014) Telomere length kinetics assay (TELKA) sorts the telomere length maintenance (tlm) mutants into functional groups. Nucleic Acids Res 42:6314-6325. doi: 10.1093/nar/gku267

Ryu T, Bonner MR, Chiolo I (2016) Cervantes and Quijote protect heterochromatin from aberrant recombination and lead the way to the nuclear periphery. Nucleus (Austin, Tex) 7:485-497. doi: 10.1080/19491034.2016.1239683

Samara NL, Datta AB, Berndsen CE, et al (2010) Structural insights into the assembly and function of the SAGA deubiquitinating module. Science 328:1025-1029. doi: 10.1126/science. 1190049

Schulze JM, Hentrich T, Nakanishi S, et al (2011) Splitting the task: Ubp8 and Ubp10 deubiquitinate different cellular pools of H2BK123. Genes Dev 25:2242-2247. doi: $10.1101 / \mathrm{gad} .177220 .111$

Schwabish MA, Struhl K (2006) Asf1 mediates histone eviction and deposition during elongation by RNA polymerase II. Mol Cell 22:415-422. doi: 10.1016/j.molcel.2006.03.014

Shachar R, Ungar L, Kupiec M, et al (2008) A systems-level approach to mapping the telomere length maintenance gene circuitry. Mol Syst Biol. doi: 10.1038/msb.2008.13

Shim EY, Ma JL, Oum JH, et al (2005) The yeast chromatin remodeler RSC complex facilitates end joining repair of DNA double-strand breaks. Mol Cell Biol 25:39343944. doi: 10.1128/MCB.25.10.3934-3944.2005

Simonicova L, Dudekova H, Ferenc J, et al (2015) Saccharomyces cerevisiae as a model for the study of extranuclear functions of mammalian telomerase. Curr Genet 61:517527. doi: 10.1007/s00294-014-0472-8

Sinclair DA, Guarente L (1997) Extrachromosomal rDNA circles--a cause of aging in yeast. Cell 91:1033-1042.

Spedale G, Timmers HTM, Pijnappel WWMP (2012) ATAC-king the complexity of SAGA during evolution. Genes Dev 26:527-541. doi: 10.1101/gad.184705.111

Sun Z-W, Allis CD (2002) Ubiquitination of histone H2B regulates H3 methylation and 
gene silencing in yeast. Nature 418:104-108. doi: 10.1038/nature00883

Titus LC, Dawson TR, Rexer DJ, et al (2010) Members of the RSC chromatin-remodeling complex are required for maintaining proper nuclear envelope structure and pore complex localization. Mol Biol Cell 21:1072-1087. doi: 10.1091/mbc.E09-07-0615

Tong XJ, Li QJ, Duan YM, et al (2011) Est1 Protects Telomeres and Inhibits Subtelomeric Y'-Element Recombination. Mol Cell Biol 31:1263-1274. doi: 10.1128/MCB.00831-10

Ungar L, Yosef N, Sela Y, et al (2009) A genome-wide screen for essential yeast genes that affect telomere length maintenance. Nucleic Acids Res 37:3840-3849. doi: $10.1093 /$ nar/gkp259

Wang L, YR Dent S (2014) Functions of SAGA in development and disease. Epigenomics 6:329-339. doi: 10.2217/epi.14.22

Wong MCVL, Scott-Drew SRS, Hayes MJ, et al (2002) RSC2, encoding a component of the RSC nucleosome remodeling complex, is essential for 2 microm plasmid maintenance in Saccharomyces cerevisiae. Mol Cell Biol 22:4218-4229. doi: 10.1128/MCB.22.12.4218-4229.2002

Wu M-Y, Lin C-Y, Tseng H-Y, et al (2017a) H2B ubiquitylation and the histone chaperone Asf1 cooperatively mediate the formation and maintenance of heterochromatin silencing. Nucleic Acids Res 45:8225-8238. doi: 10.1093/nar/gkx422

Wu Z, Liu J, Zhang Q-D, et al (2017b) Rad6-Bre1-mediated H2B ubiquitination regulates telomere replication by promoting telomere-end resection. Nucleic Acids Res 45:33083322. doi: 10.1093/nar/gkx101

Yosef N, Ungar L, Zalckvar E, et al (2009) Toward accurate reconstruction of functional protein networks. Mol Syst Biol 5:248. doi: 10.1038/msb.2009.3 
(a)

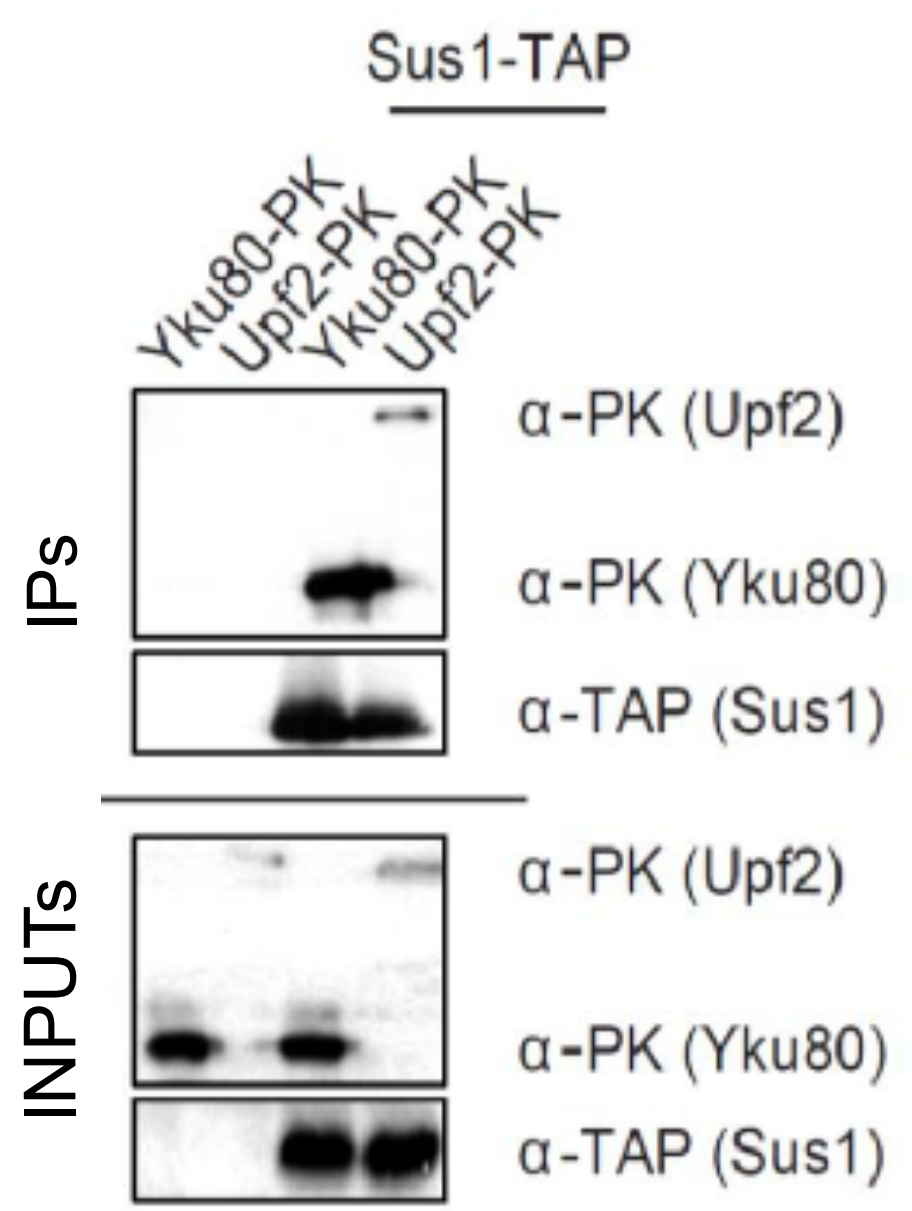

(b)

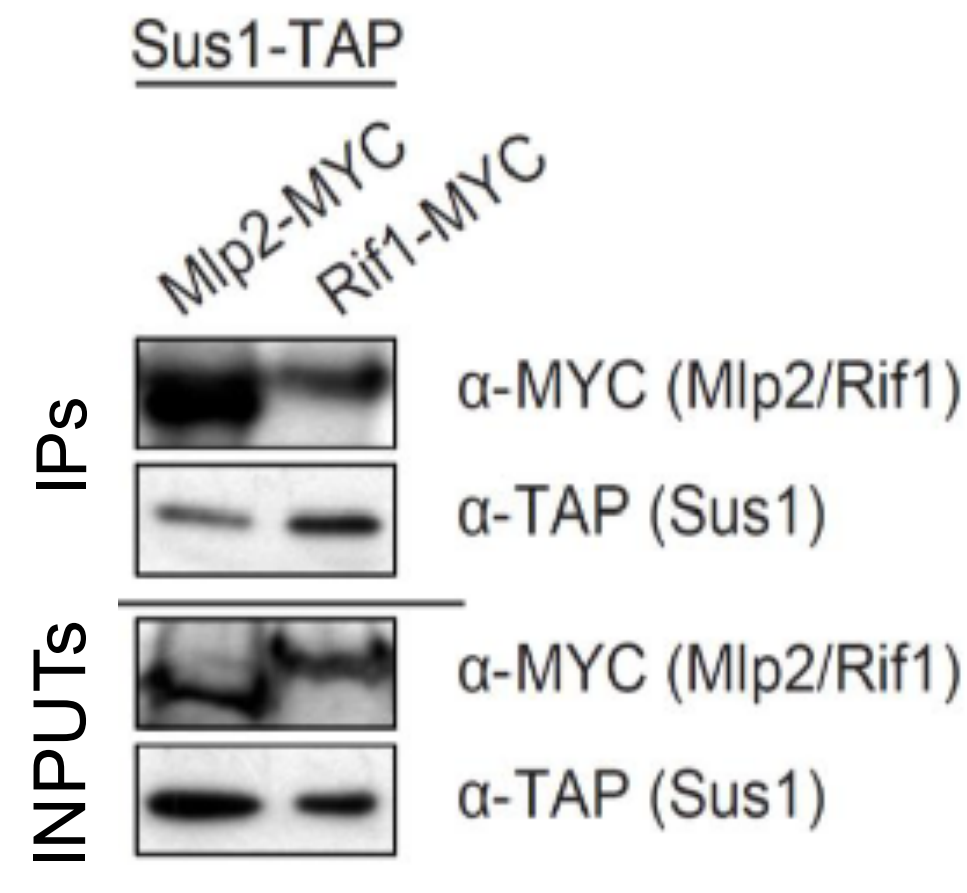

Fig. 1 Galán et al 2017 
(a)

(b)

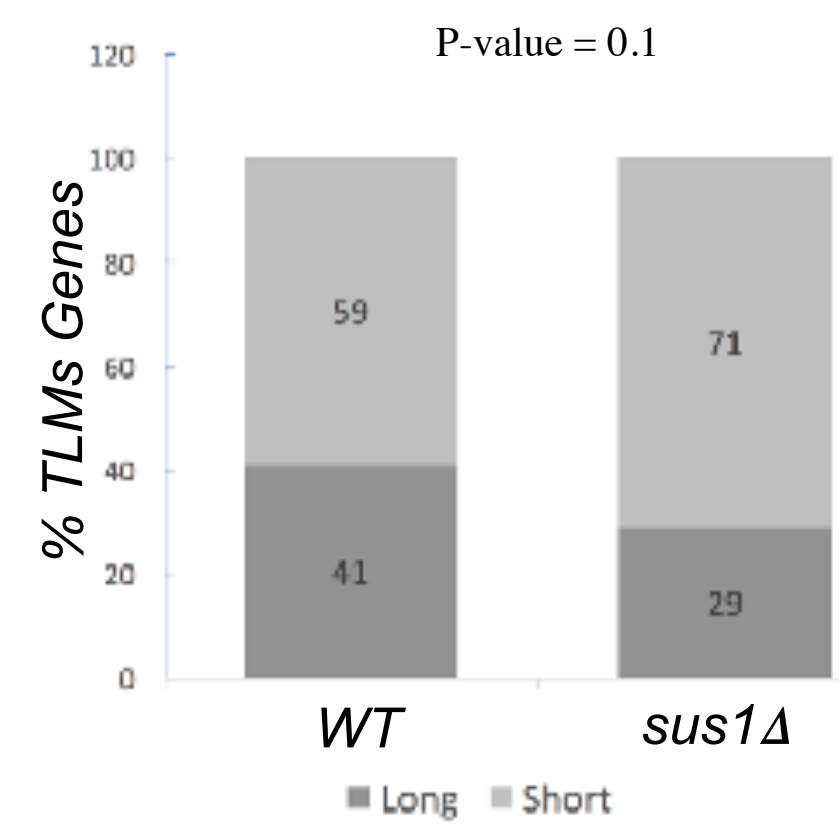

P-value $=3.889 \mathrm{e}-05$

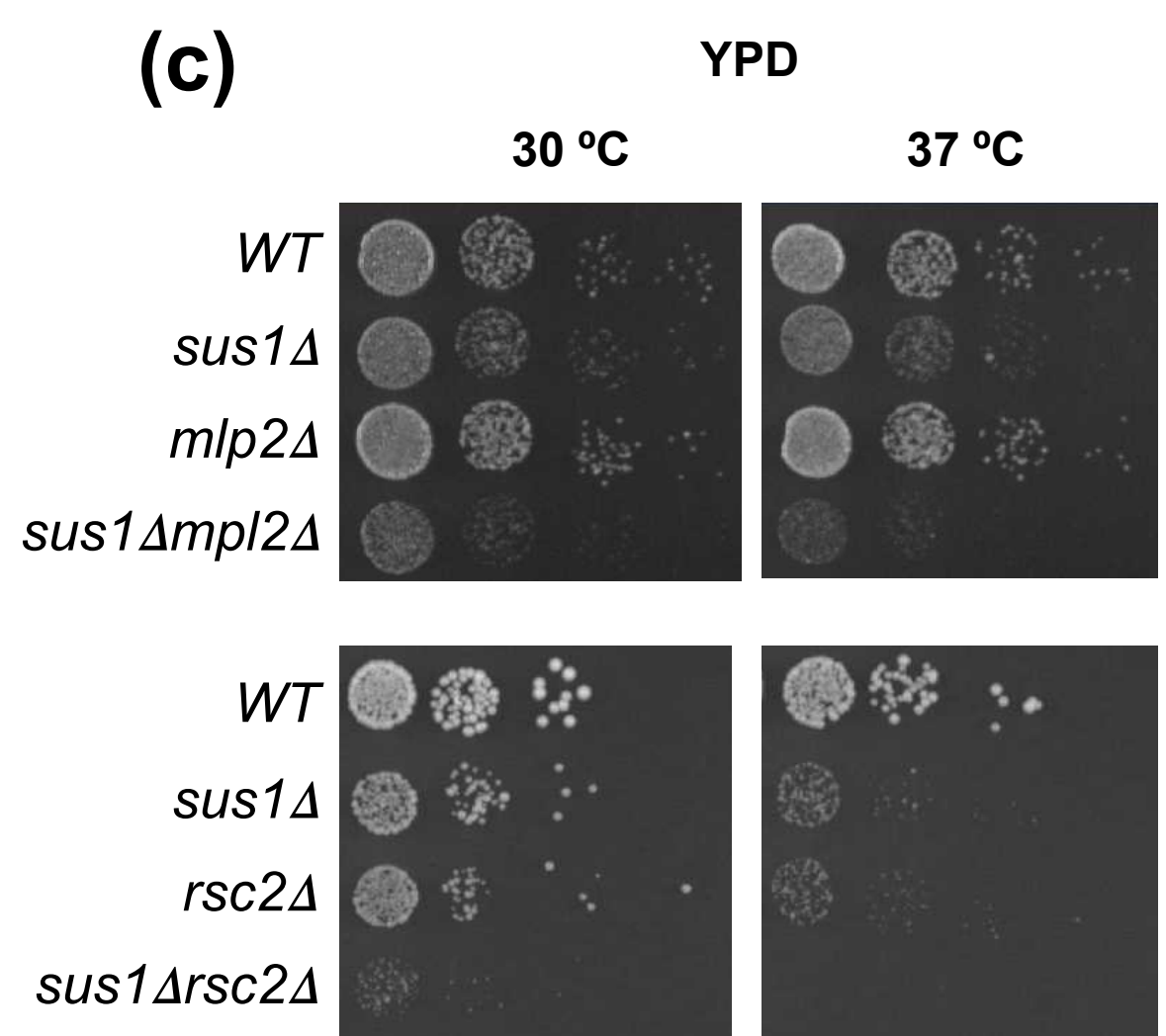

Fig. 2 Galán et al 2017 
(a)

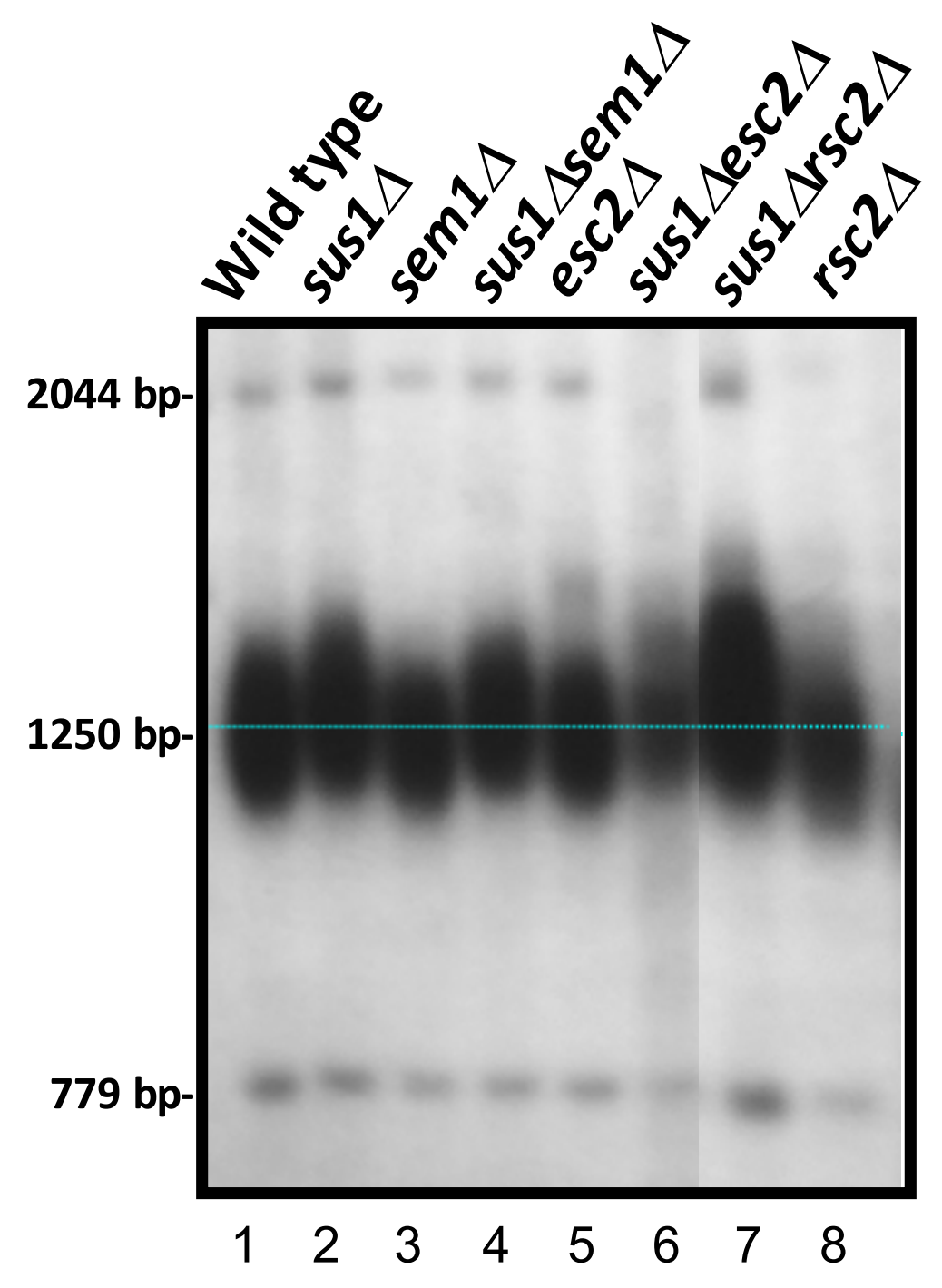

(b)

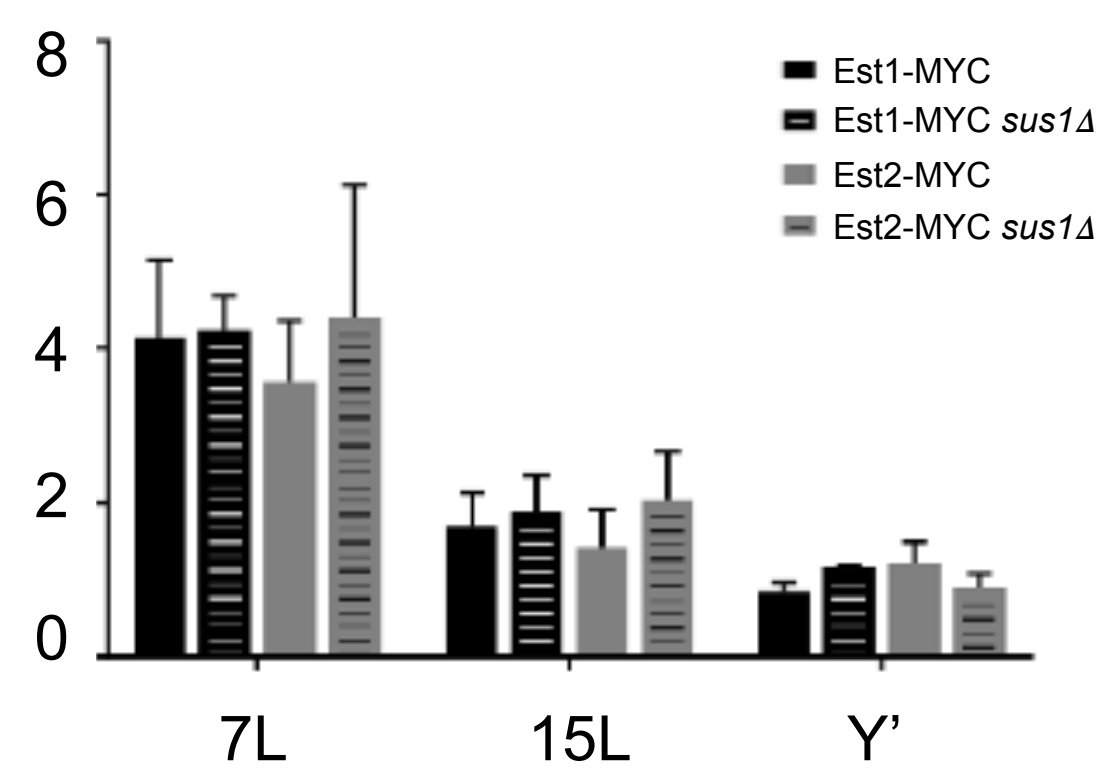

(c)

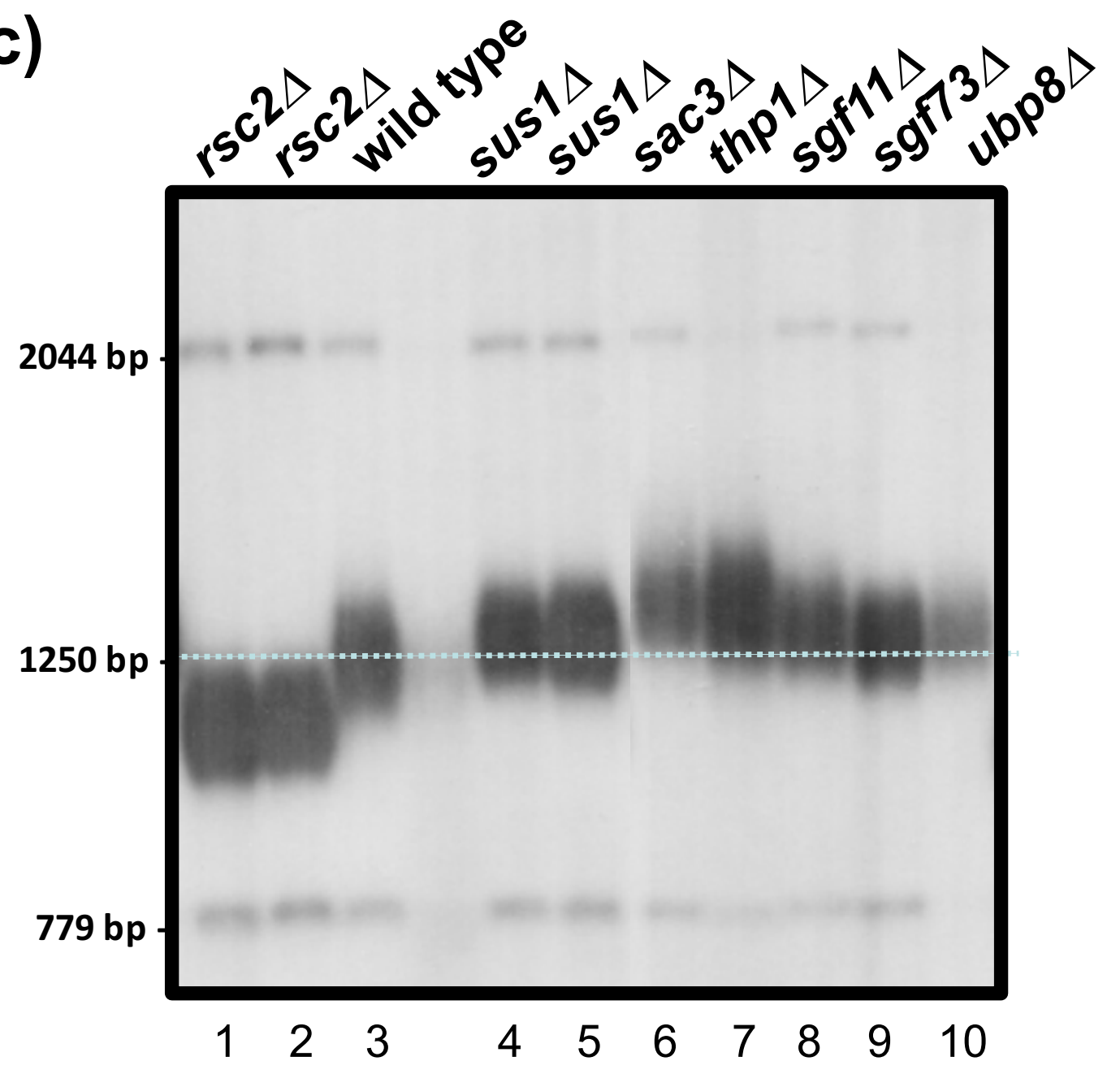

Fig. 3 Galán et al 2017 
(a)

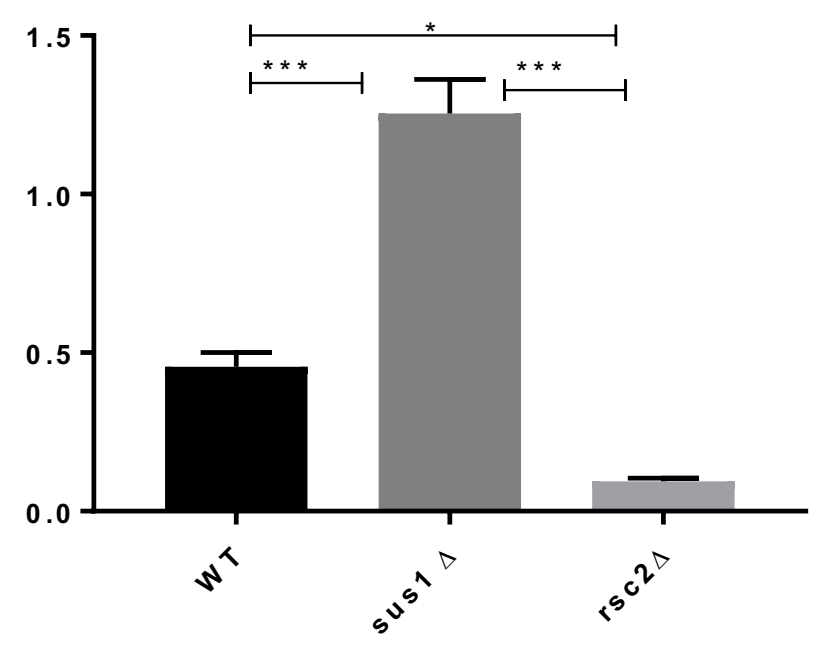

- $W T$

sus1a

$r s c 2 \Delta$

(b)

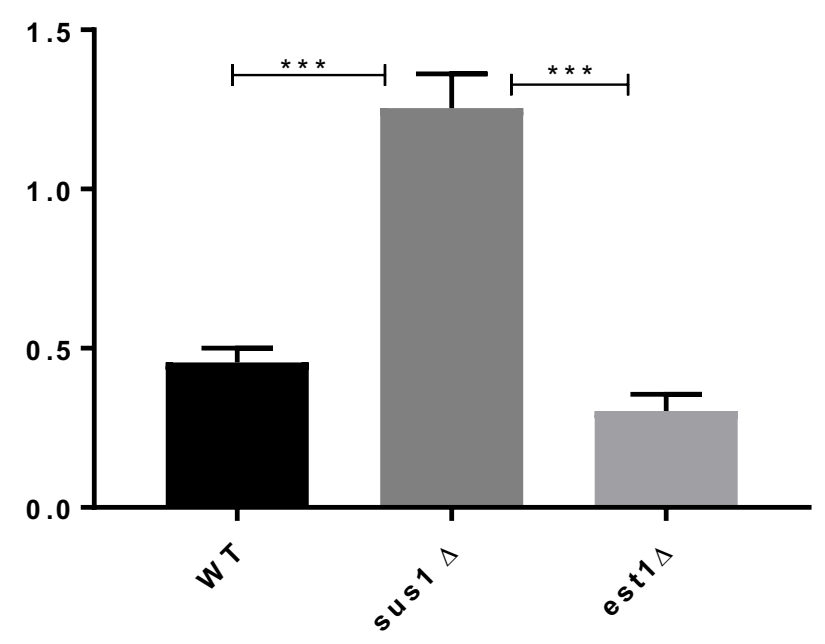

- WT

sus1a

est1

(c)
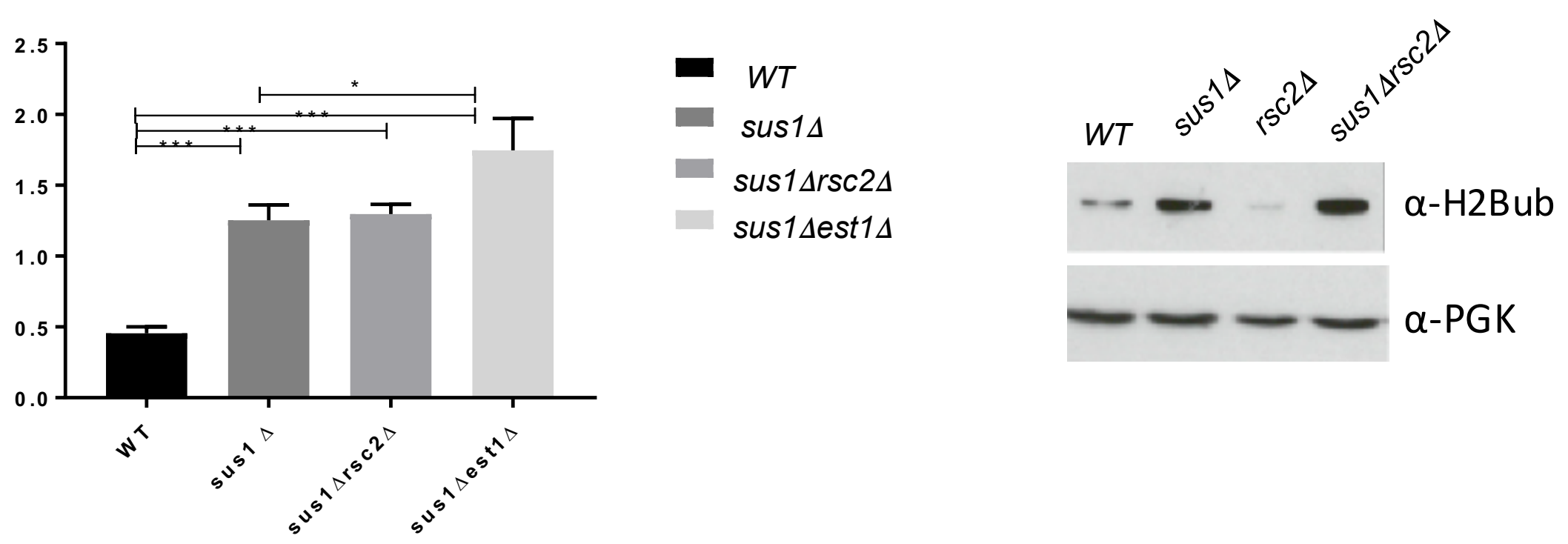

Fig. 4 Galán et al 2017 